\title{
Erratum to: Radio-protective and antioxidative activities of astaxanthin from newly isolated radio-resistant bacterium Deinococcus sp. strain WMA-LM9
}

\author{
Wasim Sajjad $^{1,2}$ • Manzoor Ahmad ${ }^{1}$ - Salman Khan ${ }^{1} \cdot$ Sunniya Ilyas $^{1} \cdot$ Fariha Hasan $^{1}$. \\ Cem Celik $^{2} \cdot$ Kerry McPhail $^{2} \cdot$ Aamer Ali Shah $^{1}$
}

Published online: 28 June 2017

(C) Springer-Verlag GmbH Germany and the University of Milan 2017

\section{Erratum to: Ann Microbiol}

DOI 10.1007/s13213-017-1269-z

The original article contained errors, correct data is given below. The original article was corrected.

The name of Manzoor Ahmad is also corrected in this article.

This sentence,

For this purpose, the DNA was extracted using a DNA extraction kit (QIAGEN, Hilden, Germany), and the 16S rRNA gene sequence was amplified using $27 \mathrm{~F}^{\prime}$ (5'-AGAGTTTGATCCTG GCTCAG-3') and 1494R' (5'-CTACGGCTACCTTGTTACGA $\left.-3^{\prime}\right)$ bacterial primers.

Should be:

For this purpose, the DNA was extracted using a DNA extraction kit (QIAGEN, Hilden, Germany), and the 16S rRNA gene sequence was amplified using 27F' (5'-AGAGTTTGATCCTG GCTCAG-3') and 1492R' (5'-CTACGGCTACCTTGTTACGA $-3^{\prime}$ ) bacterial primers.

The online version of the original article can be found at http://dx.doi.org/ 10.1007/s13213-017-1269-z

Aamer Ali Shah

alishah@qau.edu.pk

1 Department of Microbiology, Faculty of Biological Sciences, Quaid-i-Azam University, Islamabad 45320, Pakistan

2 Department of Pharmaceutical Sciences, College of Pharmacy, Oregon State University, Corvallis, OR 97331, USA
This sentence,

The carotenoid extracts from Deinococcus sp. WMA-LM9 were analysed in an liquid chromatography-tandem mass spectrometry (LC-MS/MS; Varian 1200 Triple-Quadrupole-LC-MS system; Varian, Palo Alto, CA).

Should be:

The carotenoid extracts from Deinococcus sp. WMA-LM9 were analysed in an liquid chromatography-tandem mass spectrometry (ABX3200 Q-TRAP mass spectrometer equipped with a TurbolonSpray ESI source, and connected to a Shimadzu HPLC system with dual LC-20 pumps, a SPD-M20A UV/Vis photodiode array (PDA) detector and auto sampler).

This sentence,

A nuclear magnetic resonance (NMR) spectrum provides prime evidence on the structure of a compound. NMR spectroscopic data were recorded at room temperature on a Bruker AMX $400 \mathrm{MHz}$ and AMX $600 \mathrm{MHz}$ NMR spectrometer (Bruker Corp., Billerica, MA) in dCDCl3 $(\mathrm{CDCl} 3)$, with tetramethylsilane as an internal standard.

Should be:

A nuclear magnetic resonance (NMR) spectrum provides prime evidence on the structure of a compound. NMR spectroscopic data were recorded at room temperature on a Bruker Avance $400 \mathrm{MHz}$ NMR spectrometer (Bruker Corp., Billerica, MA) in $\mathrm{dCDCl} 3(\mathrm{CDCl} 3)$ referenced to 
residual protonated solvent $(\delta 7.24 \mathrm{ppm}, \delta 77.0 \mathrm{ppm})$, with tetramethylsilane as an internal standard.

This sentence,

In the present study surface soil collected from the desert of District Lucky Marwat was screened for the isolation of radio- resistant bacteria.

Should be:

In the present study surface soil collected from the desert of District Lakki Marwat was screened for the isolation of radioresistant bacteria. 